\title{
Thrombotic vs. Bleeding Events of Interruption of Dual Antiplatelet Therapy within 12 Months among Patients with Stent-Driven High Ischemic Risk Definition following PCI
}

\author{
Hao-Yu Wang $D^{1},{ }^{1}$ Bo Xu, ${ }^{2}$ Chen-Xi Song, ${ }^{1}$ Chang-Dong Guan, ${ }^{2}$ Li-Hua Xie, ${ }^{2}$ \\ Yan-Yan Zhao, ${ }^{3}$ Zhong-Xing Cai, ${ }^{1}$ Sheng Yuan, ${ }^{1}$ and Ke-Fei Dou ${ }^{1}{ }^{1}$ \\ ${ }^{1}$ Department of Cardiology, Cardiometabolic Medicine Center, Coronary Heart Disease Center, Fuwai Hospital, \\ National Center for Cardiovascular Diseases, State Key Laboratory of Cardiovascular Disease, \\ Chinese Academy of Medical Sciences and Peking Union Medical College, Beijing, China \\ ${ }^{2}$ Catheterization Laboratories, Fuwai Hospital, National Center for Cardiovascular Diseases, \\ Chinese Academy of Medical Sciences and Peking Union Medical College, Beijing, China \\ ${ }^{3}$ Medical Research and Biometrics Center, Fuwai Hospital, National Center for Cardiovascular Diseases, \\ Chinese Academy of Medical Sciences, Peking Union Medical College, Beijing, China
}

Correspondence should be addressed to Ke-Fei Dou; drdoukefei@126.com

Received 21 July 2021; Accepted 22 December 2021; Published 13 January 2022

Academic Editor: Thach N. Nguyen

Copyright $(2022$ Hao-Yu Wang et al. This is an open access article distributed under the Creative Commons Attribution License, which permits unrestricted use, distribution, and reproduction in any medium, provided the original work is properly cited.

Background. There is a paucity of real-world data regarding the clinical impact of dual antiplatelet therapy (DAPT) interruption (temporary or permanent) among patients at high ischemic risk. The aim of this study was to assess the risk of cardiovascular events after interruption of DAPT in high-risk PCI population. Methods. This study used data from the Fuwai PCI registry, a large, prospective cohort of consecutive patients who underwent PCI. We assessed 3,931 patients with at least 1 high ischemic risk criteria of stent-related recurrent ischemic events proposed in the 2017 ESC guidelines for focused update on DAPT who were free of major cardiac events in the first 12 months. The primary ischemic endpoint was 30-month major adverse cardiac and cerebrovascular events, and the key safety endpoints were BARC class 2, 3, or 5 bleeding and net adverse clinical events. Results. DAPT interruption within 12 months occurred in 1,122 patients $(28.5 \%)$, most of which were due to bleeding events or patients' noncompliance to treatment. A multivariate Cox regression model, propensity score (PS) matching, and inverse probability of treatment weighting (IPTW) based on the propensity score demonstrated that DAPT interruption significantly increased the risk of primary ischemic endpoint compared with prolonged DAPT (3.9\% vs. 2.2\%; Cox-adjusted hazard ratio (HR): $1.840 ; 95 \%$ confidence interval (CI): 1.247 to 2.716; PS matching-HR: 2.049 [1.236-3.399]; IPTW-adjusted HR: 1.843 [1.250-2.717]). This difference was driven mainly by all-cause death ( $1.8 \%$ vs. $0.7 \%)$ and MI (1.3\% vs. $0.5 \%)$. Furthermore, the rate of net adverse clinical events (4.9\% vs. 3.2\%; Cox-adjusted HR: 1.581 [1.128-2.216]; PS matching-HR: 1.639 [1.075-2.499]; IPTW-adjusted HR: 1.554 [1.110-2.177]) was also higher in patients with DAPT interruption ( $\leq 12$ months), whereas no significant differences between groups were observed in terms of BARC 2, 3, or 5 bleeding. These findings were consistent across various stent-driven highischemic risk subsets with respect to the primary ischemic endpoints, with a greater magnitude of harm among patients with diffuse multivessel diabetic coronary artery disease. Conclusions. In patients undergoing high-risk PCI, interruption of DAPT in the first 12 months occurred infrequently and was associated with a significantly higher adjusted risk of major adverse cardiovascular events and net adverse clinical events. 2017 ESC stent-driven high ischemic risk criteria may help clinicians to discriminate patient selection in the use of long-term DAPT when the ischemic risk certainly overcomes the bleeding one. 


\section{Introduction}

Dual antiplatelet therapy (DAPT) of aspirin and a $\mathrm{P}_{12}$ inhibitor has been a therapeutic cornerstone after percutaneous coronary intervention (PCI) or acute coronary syndrome (ACS); however, its optimal duration in different clinical scenarios is currently a matter of debate [1,2]. After PCI with drug-eluting stent (DES) implantation, DAPT is generally recommended for 12 months in ACS patients and for 6 months in patients with stable coronary artery disease (CAD) [3]. Although DAPT is continued beyond 12 months after stenting to offer a broader atherothrombotic risk protection, this risk reduction comes at the cost of an increased risk of bleeding [4]. Based on lower rates of late stent thrombosis with newer-generation DES, the risk of thrombotic events is not increased even with 1 to 6 months of DAPT [5]. Many clinical trials (most of which were relatively small and open-label noninferiority trials) have suggested that the benefits of lower risk of bleeding events with abbreviated DAPT followed by aspirin-based single antiplatelet therapy (SAPT) were counterbalanced by higher rates of stent thrombosis [6-9], while an individual participant data meta-analysis showed that $\mathrm{P} 2 \mathrm{Y}_{12}$ inhibitor monotherapy after short DAPT was associated with lower major bleeding and similar risks of fatal and ischemic events compared with traditional DAPT [10].

Considering populations in the trials investigating the optimal minimal duration of DAPT followed by aspirin monotherapy mostly constituted of selected patients undergoing elective noncomplex PCI [11], limited and controversial evidence is available on the value of aspirin-based SAPT after shortened DAPT in intermediate-to-high-risk patients. Observational studies have reported increased risk of myocardial infarction (MI) and adverse cardiac outcomes for patients with DAPT interruptions within 6 months after PCI [12-15]. In that respect, the prognostic significance of interruption or any nonadherence to DAPT in the first 12 months in higher-risk routine practice populations remains unclear. Therefore, using prospective data from a contemporary real-world group of patients undergoing PCI, we focused on a subset of patients who satisfied high ischemic risk criteria based on patient-related clinical and angiographic characteristics and PCI-related features (using the 2017 ESC updates for DAPT guidelines) to estimate the incidence of DAPT interruption (temporary or permanent) in the first 12 months after PCI and evaluate the efficacy and safety of DAPT interruption $\leq 12$ months as compared with longer than 12 months of DAPT for these high-risk patients.

\section{Methods}

2.1. Study Population. The Fuwai PCI registry database, which involves prospective recruitment of consecutive patients undergoing PCI with DES placement between January 2013 to December 2013 at the Fuwai Hospital (National Center for Cardiovascular Diseases, Beijing, China), was used for the current retrospective analysis. The present analysis included patients with high ischemic risk defined by 2017 ESC DAPT guidelines who were event free at
12 months. We excluded patients who had a major adverse cardiac or cerebrovascular event (the composite of all-cause death, MI, or stroke), repeat revascularization, stent thrombosis, or Bleeding Academic Research Consortium (BARC) type 3 or 5 bleeding at 12-month follow-up. Demographic and clinical characteristics, angiographic and procedural information, and in-hospital and follow-up outcomes were systematically collected and were prospectively entered into the dedicated database. Institutional review board approval was granted for this research by the ethics committee of Fuwai hospital, and written informed consent was obtained for all participants for participation in this prospective registry. This study was conducted in accordance with the Declaration of Helsinki.

2.2. Procedures and Follow-Up. The PCI procedure, including device selection and revascularization strategy, and related management followed standard guidelines at the discretion of the treating physician $[16,17]$. Aspirin $300 \mathrm{mg}$ and a loading dose of a P2 $\mathrm{Y}_{12}$ inhibitor (clopidogrel 300 or $600 \mathrm{mg}$ or ticagrelor $180 \mathrm{mg}$ ) were given before intervention. After PCI, patients were prescribed $100 \mathrm{mg} /$ day aspirin indefinitely and $\mathrm{P}_{2} \mathrm{Y}_{12}$ inhibitor (clopidogrel $75 \mathrm{mg}$ once daily or ticagrelor $90 \mathrm{mg}$ twice daily) for 12 months. Detailed information on procedures is shown in the supplementary materials. Clinical follow-up was prospectively conducted via office visit or telephone contact at 30 days, 6 months, 12 months, and annually thereafter. At follow-up, data about patients' clinical status, all interventions received, and outcome events were documented by independent research personnel. Information regarding time of DAPT cessation, which drug (aspirin or $\mathrm{P}_{2} \mathrm{Y}_{12}$ inhibitor) was stopped, and the reason for stopping treatment was collected. Other possible follow-up information was obtained from hospital readmission, outpatient records, the referring physician and relatives, and external medical records from other hospitals, as necessary.

2.3. Definitions and Outcomes. Interruptions of DAPT within 12 months were defined as either a temporary interruption of aspirin and/or P2Y12 inhibitor (interruption of at least 1 days) or a permanent discontinuation ( $>30$ days). Permanent DAPT discontinuation was considered if DAPT was never resumed 30 days after discontinuation. Patients were defined as high ischemic risk if they met at least 1 of the following characteristics according to 2017 ESC guidelines for focused update on DAPT in CAD: diabetes with diffuse multivessel CAD, chronic kidney disease, at least 3 lesions treated, at least 3 stents implanted, a total stent length of more than $60 \mathrm{~mm}$, a bifurcation lesion treated with two stents, and treatment of chronic total occlusion [3]. Adapting the criteria to fit the available information, a subset of patients with at least 1 modified ESC high ischemic risk criteria (without information on previous stent thrombosis on antiplatelet therapy and last patent vessel) was defined.

Given that the clinical variables evaluated in the present analysis were collected at a time when the Academic Research Consortium for High Bleeding Risk (ARC-HBR) definition 
was not yet available, Supplementary Table 1 illustrates the list of major and minor ARC-HBR criteria and their respective definitions adapted to the current study database. Patients were defined as HBR if they met at least 1 major or 2 minor criteria [18]. Conversely, those not meeting any ARC-HBR criterion or patients with only 1 minor criterion were considered non-HBR.

The primary ischemic endpoint was major adverse cardiac and cerebrovascular events, defined as a composite of allcause death, MI, or stroke. The key secondary endpoint was clinically relevant bleeding defined by BARC 2-, 3-, or 5-type bleedings [19] and net adverse clinical events, defined as a composite of clinically relevant bleeding and major adverse cardiac and cerebrovascular events. Secondary end points included all-cause death, cardiac death, MI, definite or probable ST, and stroke. Cardiac mortality was defined according to Academic Research Consortium (ARC) criteria as any death because of an immediate cardiac cause, deaths related to the procedure, or undetermined cause of death [20]. The diagnosis of MI was based on the Third Universal Definition of MI [21]. Stent thrombosis was defined as definite or probable stent thrombosis based on the ARC classification [20]. Stroke was defined as a focal loss of neurologic function caused by an ischemic or hemorrhagic event, with residual symptoms lasting at least 24 hours or leading to death [22]. Source documents were obtained for any adverse events or any DAPT cessation. All clinical events were adjudicated by an independent clinical event committee, composed of members who did not participate in patient enrollment for this study. All endpoints were evaluated at 30 months. Median follow-up was 877 days (interquartile range: 808 to 944 days).

2.4. Statistical Analysis. Categorical data are described as frequency and percentages and continuous data as means with standard deviations. Statistical significance of differences in continuous variables between patient groups was tested with the use of two-sample Student's $t$-test; the chisquare or Fisher exact test was used for categorical variables. Time-to-event data were plotted using the Kaplan-Meier method and compared using the log-rank test. In an attempt to reduce the impact of treatment selection bias inherent to an observational study, three sensitivity analyses were performed to adjust for confounding factors as much as possible. First, a multivariable Cox proportional hazard regression model was used to estimate the independent effect of DAPT duration on clinical outcome. Factors included in multivariable models were based on variables with $P<0.10$ in the univariate analyses, along with traditional cardiac risk factors. The variables selected appear in the full model shown in Table 1. Second, the propensity score matching (PSM) method was performed. Propensity scores were created by a multivariable logistic regression model with the dependent variable of DAPT duration and a list of covariates as the independent variables. We matched patients with prolonged DAPT ( $>12$-month) to those with discontinued DAPT within 12 months using the $1: 1$ nearest neighbor approach without replacement with a caliper width of $0.2 \mathrm{SD}$ of the logit of the propensity score. Variables included in the PSM models are presented in Supplementary Table 2. Third, we used the inverse probability of treatment weighting (IPTW) Cox proportional hazard regression model to estimate the average treatment effects. In this approach, the weights for patients treated with interruptions ( $\leq 12$ months) of DAPT were the inverse of (1-propensity score), and the weights for patients treated with DAPT $>12$ months were the inverse of propensity score. Balance between the two groups after PSM and IPTW was evaluated by the standardized difference, using a threshold of less than $10 \%$ to indicate a balance. Sensitivity analysis was performed with the entire population $(n=4,430)$, including patients who were followed up for $<12$ months or presented with adverse events within 12 months after PCI in patients who satisfied high ischemic risk criteria. The consistency of effects on primary ischemic endpoint was also explored across the individual components of the ESC high ischemic risk definition, the number of high ischemic risk criteria fulfilled (1, 2 , or 3 or more features), and major subgroups. $P<0.05$ was considered significant for all analyses. Statistical analyses were conducted using SPSS version 24 (SPSS Inc., Chicago, IL, USA) and R version 3.2.0 (R Foundation for Statistical Computing, Vienna, Austria).

\section{Results}

3.1. Cohort Characteristics. A total of 10,167 consecutive patients undergoing PCI with DES were eligible for evaluation. Based on the adapted high ischemic risk criteria defined by the 2017 ESC DAPT guidelines, 4,430 patients satisfied at least 1 criterion and were, thus, considered to be at high ischemic risk. Of these, 479 patients with adverse clinical events during the 12-month follow-up after PCI and 20 patients with incomplete follow-up on clinical outcomes within 12 months were excluded. The final cohort consisted of 3,931 patients who were at high ischemic risk and survived the first year after PCI without a major ischemic or bleeding event, of whom $1,122(28.5 \%)$ had an interruption or discontinued DAPT in the first 12 months (Figure 1). Source documents indicated BARC type 1 or 2 bleeding as the most commonly identified reason for the DAPT cessation (42\%), followed by nonadherence $(31 \%)$, need for surgery $(12 \%)$, other specified reasons $(6 \%)$, and unknown $(9 \%)$.

Mean patient age was 59.1 years; $76.5 \%$ were men, $47.2 \%$ had diabetes mellitus, and $57.1 \%$ presented with acute coronary syndromes (ACS). Nearly $90 \%$ of patients had multivessel CAD, and the left main or left anterior descending artery lesion was treated in about $85 \%$ of patients. The mean number of ESC high ischemic risk criteria per patient was 2.0; a total of 2,188 of 3,931 patients (55.7\%) met 2 or more criteria. The most common high ischemic risk qualifying features were at least 3 stents implanted and diffuse multivessel diabetic CAD patients (Figure 2). Patientlevel clinical, angiographic and procedural data in the DAPT interruption $\leq 12$ months and DAPT maintenance $>12$ months groups are summarized in Tables 2 and 3. Patients with DAPT interruption $\leq 12$ months more often had ACS as the indication for PCI and had higher rates of ARC for High 


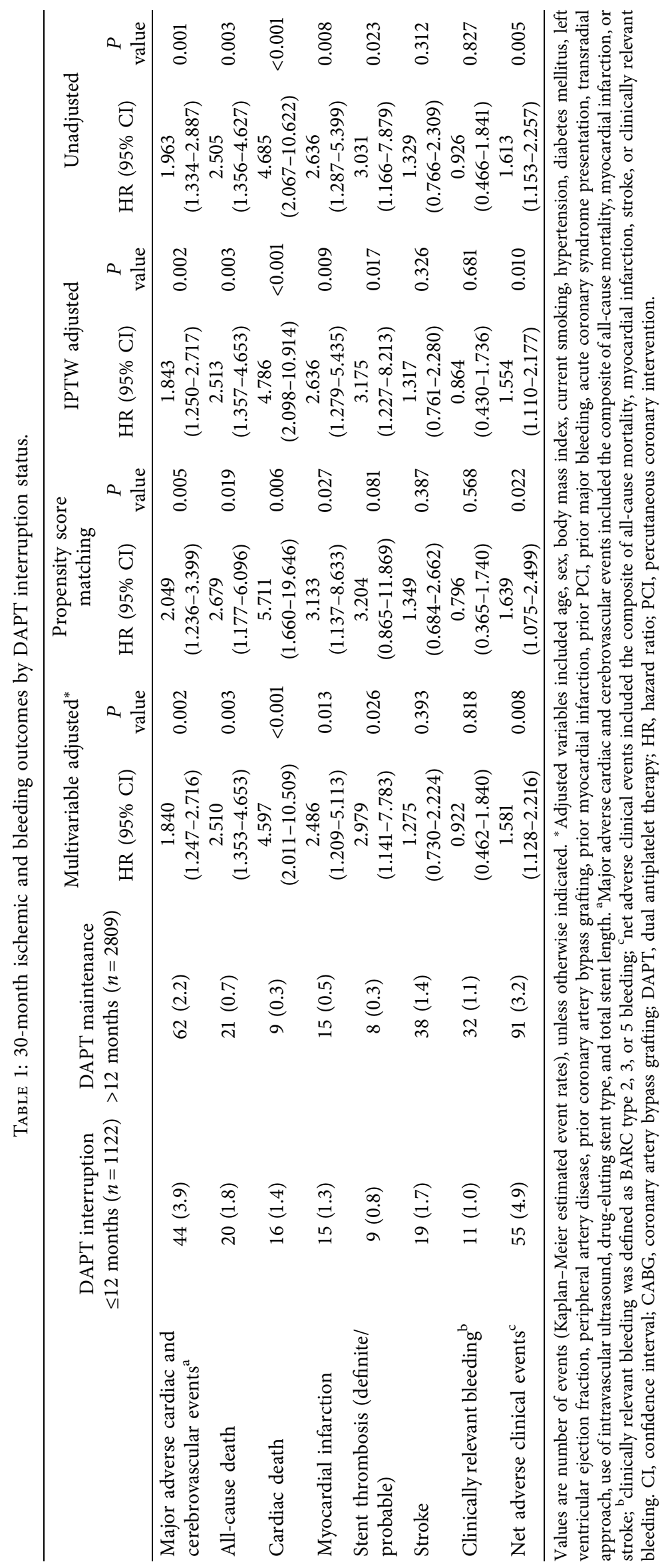




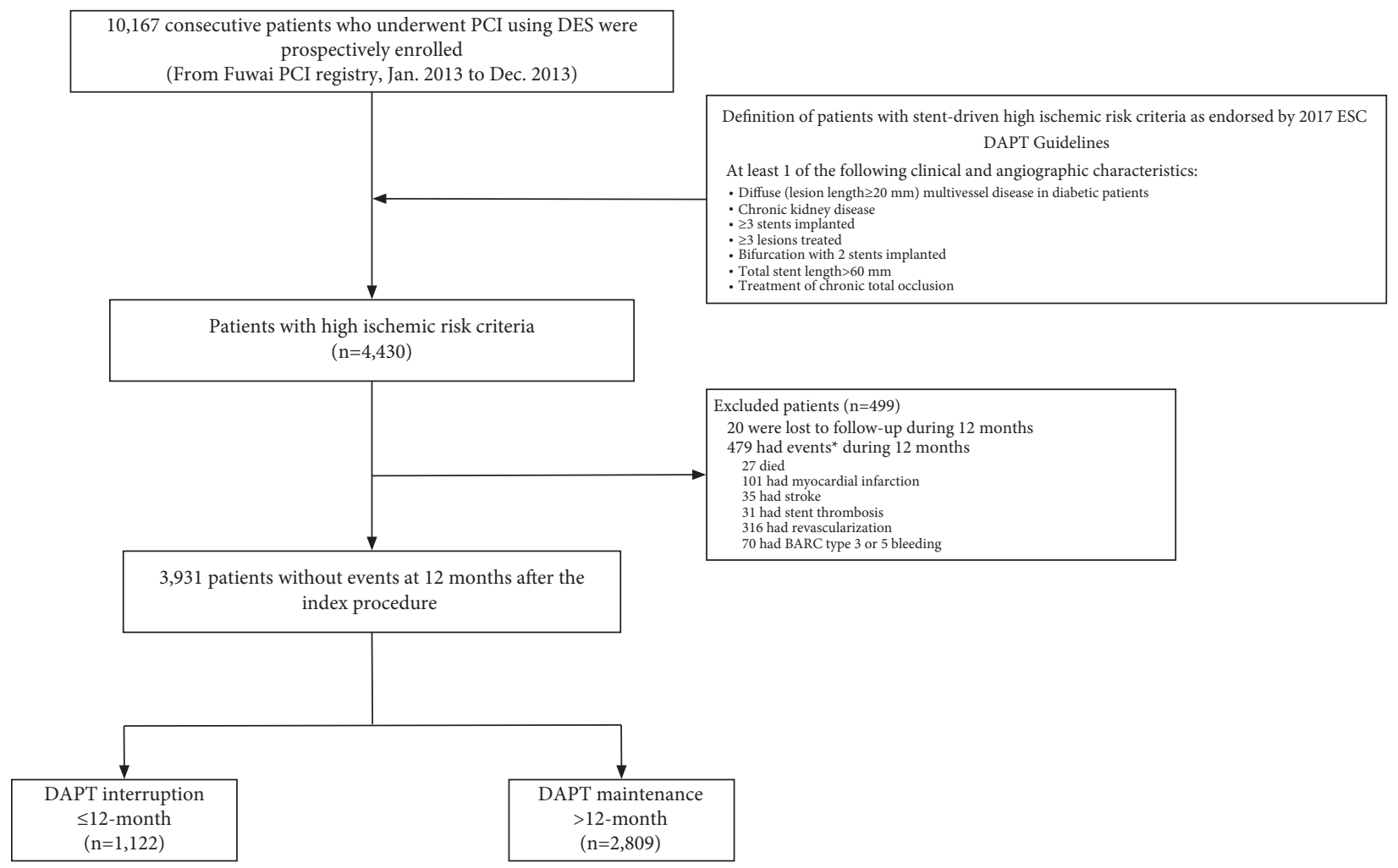

FIgure 1: Study flow diagram. BARC=Bleeding Academic Research Consortium; DES=drug-eluting stent; DAPT =dual antiplatelet therapy; ESC = European Society of Cardiology; HIR = high ischemic risk; and PCI = percutaneous coronary intervention. * Subjects may have $>1$ event.

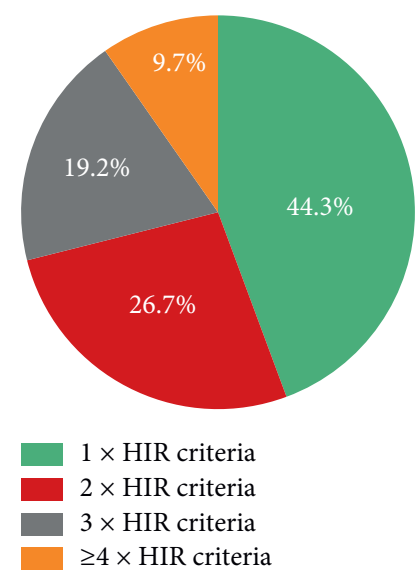

(a)

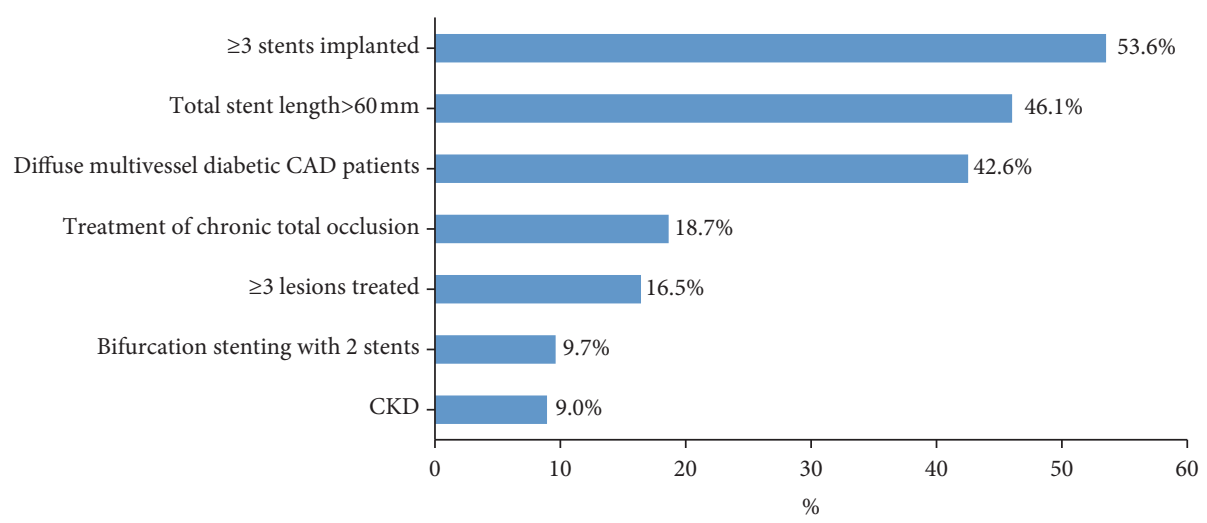

(b)

FIGURE 2: The distribution and prevalence of the ESC stent-driven high ischemic risk criteria components among patients fulfilling high ischemic risk definition. (a) The sum of high ischemic risk criteria satisfied by each patient was used to stratify patients according to the number of times they fulfilled the 2017 ESC DAPT guideline stent-driven high ischemic risk definition. The pie chart shows the distribution of HIR patients with increasing numbers of multiple coexisting criteria $(1 \times \mathrm{HIR}$ to $\geq 4 \times \mathrm{HIR})$. (b) Bars indicate the overall prevalence of each high ischemic risk criterion among patients qualified as being at high ischemic risk. HIR $=$ high ischemic risk. Other abbreviations are as in Figure 1.

Bleeding Risk (ARC-HBR) compared with DAPT $>12$ months. After PSM and IPTW, the absolute standardized differences for all the baseline patient characteristics between the two groups were less than 0.1 (Supplementary Table 2).
3.2. Impact of ESC High Ischemic Risk Criteria on Very Late Clinical Events (12 to 30 Months after PCI). At 12 to 30 months after stenting, the rate of major adverse cardiac and cerebrovascular events was higher in patients with versus without ESC high ischemic risk criteria $(2.7 \%$ vs. 
TABLE 2: Baseline characteristics.

\begin{tabular}{|c|c|c|c|}
\hline & \multicolumn{2}{|c|}{$\begin{array}{l}\text { Interruption of DAPT within } \\
12 \text { months after PCI }\end{array}$} & \multirow{2}{*}{$\begin{array}{c}P \\
\text { value }\end{array}$} \\
\hline & No $(n=2809)$ & Yes $(n=1122)$ & \\
\hline Age, years & $59.05 \pm 10.00$ & $59.33 \pm 10.48$ & 0.445 \\
\hline Male & $2155(76.7)$ & $854(76.1)$ & 0.687 \\
\hline Hyperlipidemia & $1954(69.6)$ & $751(66.9)$ & 0.108 \\
\hline Hypertension & $1926(68.6)$ & $764(68.1)$ & 0.773 \\
\hline Diabetes mellitus & $1309(46.6)$ & $545(48.6)$ & 0.263 \\
\hline Chronic kidney disease* & $248(8.8)$ & $104(9.3)$ & 0.662 \\
\hline Current smoker & $1601(57.0)$ & $627(55.9)$ & 0.525 \\
\hline Heart failure & $69(2.5)$ & $22(2.0)$ & 0.351 \\
\hline Peripheral artery disease & $89(3.2)$ & $38(3.4)$ & 0.727 \\
\hline $\begin{array}{l}\text { History of myocardial } \\
\text { infarction }\end{array}$ & $607(21.6)$ & 240 & 0.880 \\
\hline Prior PCI & $630(22.4)$ & $256(22.8)$ & 0.792 \\
\hline Prior $\mathrm{CABG}$ & $139(4$ & 53 & 0.768 \\
\hline History of stroke & $331(1$ & 152( & 0.128 \\
\hline $\begin{array}{l}\text { History of major } \\
\text { bleeding }^{\dagger}\end{array}$ & $17(0.6)$ & 12 & 0.124 \\
\hline Body mass index, $\mathrm{kg} / \mathrm{m}^{2}$ & $26.10 \pm 3.14$ & $25.97 \pm 3.28$ & 0.279 \\
\hline LVEF, \% & $62.42 \pm$ & $62.42 \pm$ & 0.991 \\
\hline Clinical presentation & & & 0.010 \\
\hline $\begin{array}{l}\text { Stable coronary artery } \\
\text { disease }\end{array}$ & $1241(44.2)$ & $445(39.7)$ & \\
\hline $\begin{array}{l}\text { Acute coronary } \\
\text { syndrome }\end{array}$ & $1568(55.8)$ & $677(60.3)$ & \\
\hline UA/NSTEMI & $1234(43.9)$ & $527(46.9)$ & 0.084 \\
\hline STEMI & $334(11.9)$ & $150(13.4)$ & 0.203 \\
\hline $\begin{array}{l}\text { White blood cell count, } \\
10^{9} / \mathrm{L}\end{array}$ & $6.80 \pm 1.6$ & $6.84 \pm 1.63$ & 0.482 \\
\hline Hemoglobin, g/dL & $14.25 \pm 1.58$ & $14.18 \pm 1.56$ & 0.220 \\
\hline Platelet count, $10^{9} / \mathrm{L}$ & $204.08 \pm 53.91$ & $205.22 \pm 53.21$ & 0.550 \\
\hline ARC-HBR & $573(20.4)$ & $264(23.5)$ & 0.032 \\
\hline \multicolumn{4}{|l|}{ Discharge medication } \\
\hline Aspirin & $2778(98.9)$ & $1112(99.1)$ & 0.554 \\
\hline Clopidogrel & $2766(98.5)$ & $1110(98.9)$ & 0.266 \\
\hline Ticagrelor & $9(0.3)$ & $8(0.7)$ & 0.107 \\
\hline$\beta$-blocker & $2584(92.0)$ & $1033(92.1)$ & 0.935 \\
\hline ACEI/ARB & $1731(61.6)$ & $694(61.9)$ & 0.893 \\
\hline $\mathrm{CCB}$ & $1408(50.1)$ & $550(49.0)$ & 0.531 \\
\hline Statin & $2701(96.2)$ & $1068(95.2)$ & 0.168 \\
\hline
\end{tabular}

Values are mean \pm SD for continuous variables and $n$ (\%) for categorical variables. ACS indicates acute coronary syndrome; ACEI, angiotensinconverting enzyme inhibitors; ARB, angiotensin II receptor antagonists; ARC-HBR, Academic Research Consortium-High Bleeding Risk; CCB, calcium channel blockers; CABG, coronary artery bypass grafting; DAPT, dual antiplatelet therapy; LVEF, left ventricular ejection fraction; NSTEMI, non-ST-segment elevation myocardial infarction; PCI, percutaneous coronary intervention; STEMI, ST-segment elevation myocardial infarction; and UA, unstable angina. ${ }^{*}$ Chronic kidney disease was defined as an estimated glomerular filtration rate of less than $60 \mathrm{~mL} / \mathrm{min} / 1.73 \mathrm{~m}^{2}$ of body surface area. ${ }^{\dagger}$ Spontaneous (nonintracranial) bleeding requiring hospitalization or transfusion.

1.6\%; adjusted HR: 1.533, 95\% CI: $1.150-2.044, P=0.004)$, a difference driven by higher rates of all-cause death $(1.0 \%$ vs. $0.6 \%)$, MI ( $0.8 \%$ vs. $0.5 \%)$, and stroke ( $1.5 \%$ vs. $0.6 \%)$ (Supplementary Table 3). Very late cardiac death was more frequent in patients with versus without ESC high ischemic risk criteria ( $0.6 \%$ vs. $0.3 \%$; HR: $2.405,95 \%$ CI: $1.250-4.626$, $P=0.009$ ). There were no significant differences in the rates of 12 - to 30-month clinically relevant bleeding between two groups. After multivariate adjustment, the presence of ESC high ischemic risk remained independently associated with increased 30-month risks for major adverse cardiac and cerebrovascular events, cardiac death, and stroke, with a trend toward an increased risk for MI and stent thrombosis.

3.3. Major Adverse Coronary Events and Safety According to the DAPT Treatment Strategy (12 to 30 Months after PCI). Among patients who satisfied high ischemic risk criteria using the 2017 ESC updates for DAPT guidelines, DAPT interruption $\leq 12$ months, compared with extended-term (>12 months) DAPT, had higher crude 30-month rates of major adverse cardiac and cerebrovascular events, all-cause death, cardiac death, MI, and stent thrombosis with similar rates of BARC-defined bleeding type 2, 3, or 5, thereby resulting in an increase in the net adverse clinical events (Figure 3). After multivariable adjustment, cessation of DAPT within 12 months significantly increased the 30month risk of major adverse cardiac and cerebrovascular events when compared with continued DAPT beyond 12 months (adjusted HR: 1.840, 95\% CI: 1.247-2.716; $P=0.002$; Table 1 and Supplementary Table 4), whereas there were no statistically significant differences in BARC 2-, 3 -, or 5-type bleedings between the 2 groups (adjusted HR: $0.922,95 \%$ CI: $0.462-1.840 ; P=0.818)$. Similar trends were observed for cardiac death (adjusted HR: 4.597, 95\% CI: 2.011-10.509; $P<0.001$ ), MI (adjusted HR: 2.486, 95\% CI: 1.209-5.113; $P=0.013$ ), and stent thrombosis (adjusted HR: 2.979, 95\% CI: 1.141-7.783; $P=0.026)$. The net adverse clinical events occurred in 91 patients $(3.2 \%)$ who received DAPT maintenance $>12$ months and in 55 patients $(4.9 \%)$ who received DAPT interruption $\leq 12$ months (adjusted HR: 1.581, 95\% CI: $1.128-2.216 ; P=0.008)$.

3.4. Sensitivity and Subgroup Analyses. Consistent results were observed when sensitivity analyses using PSM and IPTW were conducted. In the PSM and IPTW propensity score-adjusted cohort, interruption of DAPT within 12 months remained independently associated with increased risks for primary ischemic endpoint (matched HR: 2.049, 95\% CI: 1.236-3.399; IPTW-HR: 1.843, 95\% CI: 1.250-2.717), cardiac death (matched HR: 5.711, 95\% CI: 1.660-19.646; IPTW-HR: 4.786, 95\% CI: 2.098-10.914), MI (matched HR: 3.133, 95\% CI: 1.137-8.633; IPTW-HR: 2.636, 95\% CI: 1.279-5.435), and stent thrombosis (matched HR: 3.204, 95\% CI: 0.865-11.869; IPTW-HR: 3.175, 95\% CI: 1.227-8.213). There was no significant association between DAPT interruption and BARC type 2, 3, or 5 bleeding (matched HR: 0.796, 95\% CI: 0.365-1.740; IPTW-HR: 0.864, 95\% CI: 0.430-1.736). A higher risk of net adverse clinical events was identified in subjects with interruptions compared with subjects without (matched HR: 1.639, 95\% CI: 1.075-2.499; IPTW-HR: 1.554, 95\% CI: 1.110-2.177). Sensitivity analyses conducted for the entire population with stent-driven high ischemic risk definition $(n=4,430)$ exhibited consistent results for the primary and secondary endpoints (Supplementary Tables 5-7), confirming the robustness of the primary analysis. In the entire study 
TABLE 3: Lesion and procedural characteristics.

\begin{tabular}{|c|c|c|c|}
\hline & \multicolumn{2}{|c|}{$\begin{array}{l}\text { Interruption of DAPT within } 12 \text { months after } \\
\text { PCI }\end{array}$} & \multirow[t]{2}{*}{$P$ value } \\
\hline & No $(n=2809)$ & Yes $(n=1122)$ & \\
\hline \multicolumn{4}{|l|}{ Lesion characteristics } \\
\hline Multivessel CAD & $2525(89.9)$ & $998(88.9)$ & 0.382 \\
\hline \multicolumn{4}{|l|}{ Location of the lesion treated } \\
\hline LM & $140(5.0)$ & $50(4.5)$ & 0.486 \\
\hline LAD & $2386(84.9)$ & $964(85.9)$ & 0.436 \\
\hline $\mathrm{LCx}$ & $835(29.7)$ & $348(31.0)$ & 0.426 \\
\hline RCA & $959(34.1)$ & $371(33.1)$ & 0.520 \\
\hline Bypass graft & $7(0.2)$ & $3(0.3)$ & 0.919 \\
\hline \multicolumn{4}{|l|}{ Target lesion morphology } \\
\hline Heavy calcified lesion & $143(5.1)$ & $55(4.9)$ & 0.807 \\
\hline In-stent restenosis lesion & $128(4.6)$ & $49(4.4)$ & 0.796 \\
\hline Bifurcation lesion & $569(20.3)$ & $221(19.7)$ & 0.693 \\
\hline Bifurcation with two stents implanted & $283(10.1)$ & $98(8.7)$ & 0.200 \\
\hline Thrombotic lesion & $116(4.1)$ & $52(4.6)$ & 0.480 \\
\hline Chronic total occlusion & $541(19.3)$ & $194(17.3)$ & 0.153 \\
\hline Type B2 or C lesion & $2510(89.4)$ & $1020(90.9)$ & 0.146 \\
\hline SYNTAX score & $14.67 \pm 8.46$ & $14.51 \pm 8.26$ & 0.629 \\
\hline Total lesion length, mm & $57.22 \pm 30.35$ & $55.76 \pm 27.88$ & 0.163 \\
\hline \multicolumn{4}{|l|}{ Procedural characteristics } \\
\hline Number of vessels treated & $1.49 \pm 0.60$ & $1.50 \pm 0.59$ & 0.566 \\
\hline Number of lesions treated & $1.75 \pm 0.81$ & $1.75 \pm 0.81$ & 1.000 \\
\hline 1 & $1241(44.1)$ & $501(44.7)$ & 0.787 \\
\hline 2 & $1111(39.6)$ & $432(38.5)$ & 0.543 \\
\hline$\geq 3$ & $458(16.3)$ & $189(16.8)$ & 0.680 \\
\hline Number of stents implanted & $2.66 \pm 1.16$ & $2.59 \pm 1.13$ & 0.105 \\
\hline 1 & $429(15.3)$ & $189(16.8)$ & 0.221 \\
\hline 2 & $863(30.7)$ & $343(30.6)$ & 0.926 \\
\hline$\geq 3$ & $1517(54.0)$ & $590(52.6)$ & 0.420 \\
\hline Total stent length, mm & $61.00 \pm 29.34$ & $59.99 \pm 27.90$ & 0.327 \\
\hline Total stent length $>60 \mathrm{~mm}$ & $1280(45.9)$ & $523(46.6)$ & 0.666 \\
\hline Mean stent diameter, $\mathrm{mm}$ & $2.92 \pm 0.52$ & $2.91 \pm 0.54$ & 0.640 \\
\hline Vascular access site & & & 0.459 \\
\hline Radial approach & $2538(90.4)$ & $1005(89.6)$ & \\
\hline Femoral approach & $271(9.6)$ & $117(10.4)$ & \\
\hline Use of intravascular ultrasound & $214(7.6)$ & $91(8.1)$ & 0.602 \\
\hline Use of glycoprotein IIb/IIIa inhibitors & $520(18.5)$ & $223(19.9)$ & 0.324 \\
\hline Drug-eluting stent type & & & 0.949 \\
\hline First-generation DES & $286(10.2)$ & $115(10.2)$ & \\
\hline Second-generation DES & $2523(89.8)$ & $1007(89.8)$ & \\
\hline
\end{tabular}

Values are mean \pm SD for continuous variables and $n(\%)$ for categorical variables. CAD indicates coronary artery disease; DES, drug-eluting stent; LM, left main coronary artery; LAD, left anterior descending coronary artery; LCx, left circumflex coronary artery; RCA, right coronary artery; and SYNTAX, Synergy between PCI with Taxus and Cardiac Surgery.

population, interruption of DAPT in the first 12 months after PCI was associated with a significantly higher adjusted risk of not only primary ischemic outcome but also the clinically relevant bleeding and net adverse clinical composite outcomes over a 30-month period.

The differential effect of temporary or permanent DAPT interruption within the first 12 months after PCI compared with DAPT maintenance $>12$ months on primary ischemic and key secondary endpoints is shown in Figure 4 and Supplementary Figure 1. In the patients that were event free after the first year $(n=3,931)$, in contrast to patients who remained on DAPT, the adjusted HR for major adverse cardiac and cerebrovascular events and due to temporary DAPT interruption was $1.556 \quad(95 \%$ CI $0.666-3.633$;
$P=0.307)$ and to permanent DAPT discontinuation was $1.906(1.269-2.864 ; P=0.002)$ (Figure 4(a)). In supplementary analyses, the entire study population $(n=4,430)$ yielded associations for temporary and permanent DAPT discontinuation that were qualitatively similar in direction and magnitude with our overall findings (Supplementary Figure 1(a)). A similar pattern was observed for net adverse clinical events (Figure 4(c) and Supplementary Figure 1(c)). For clinically relevant bleeding events, patients who had temporary and permanent DAPT discontinuations were not associated with increased risk of major bleeding between 12 and 30 months (Figure 4(b)), while major bleeding throughout the 30-month follow up period was increased only after DAPT interruption on a permanent ( $>30$ days) 


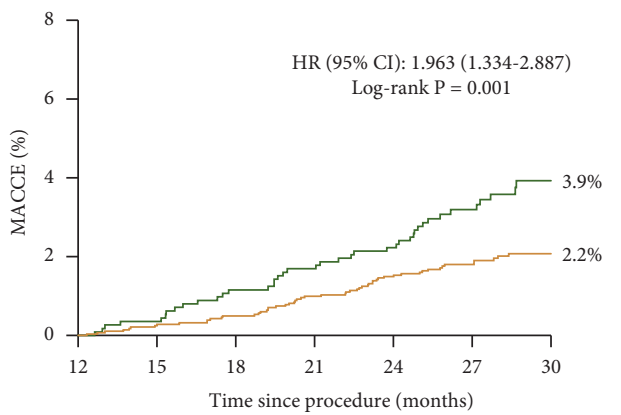

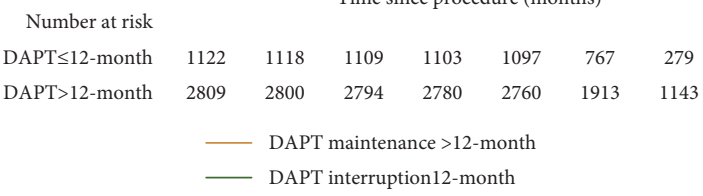

(a)

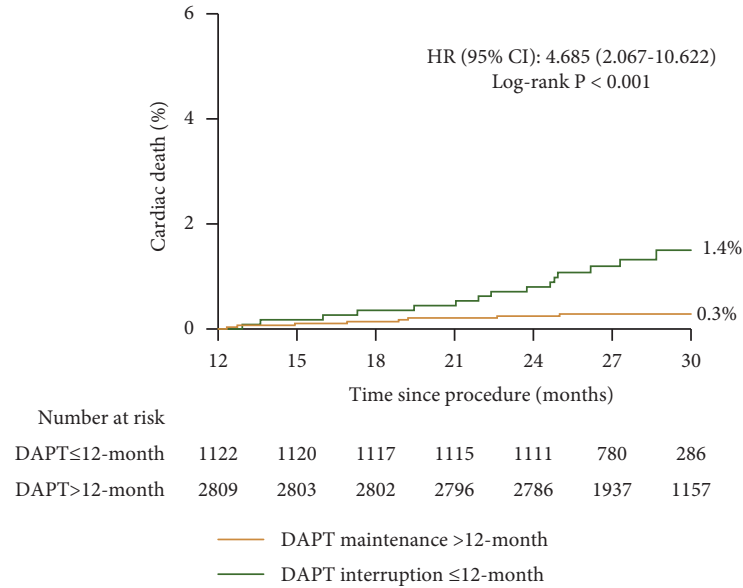

(c)

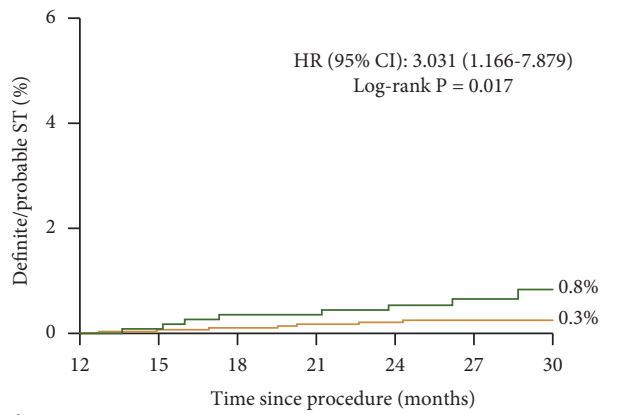

Number at risk $\begin{array}{llllllll}\text { DAPT } \leq 12-m o n t h & 1122 & 1120 & 1116 & 1114 & 1109 & 778 & 284\end{array}$ $\begin{array}{llllllll}\text { DAPT }>12-\text { month } & 2809 & 2803 & 2802 & 2794 & 2784 & 1934 & 1155\end{array}$

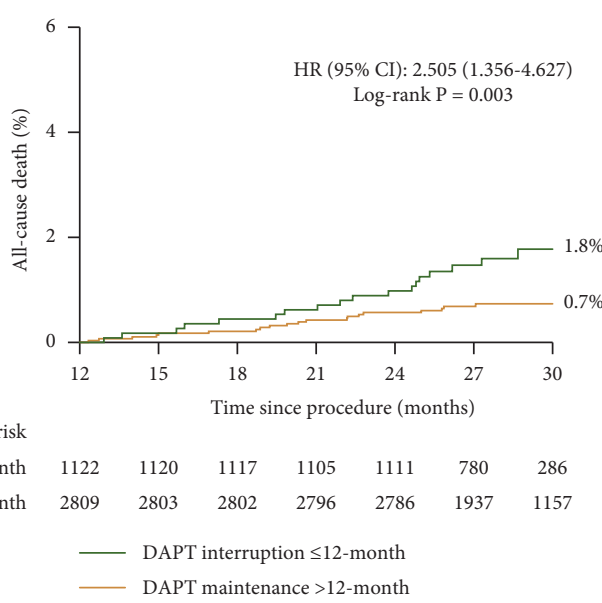

(b)

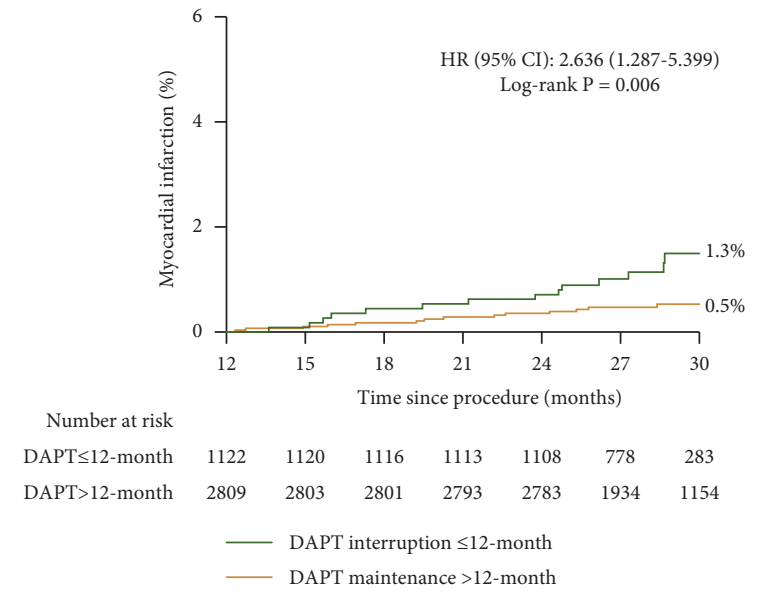

(d)

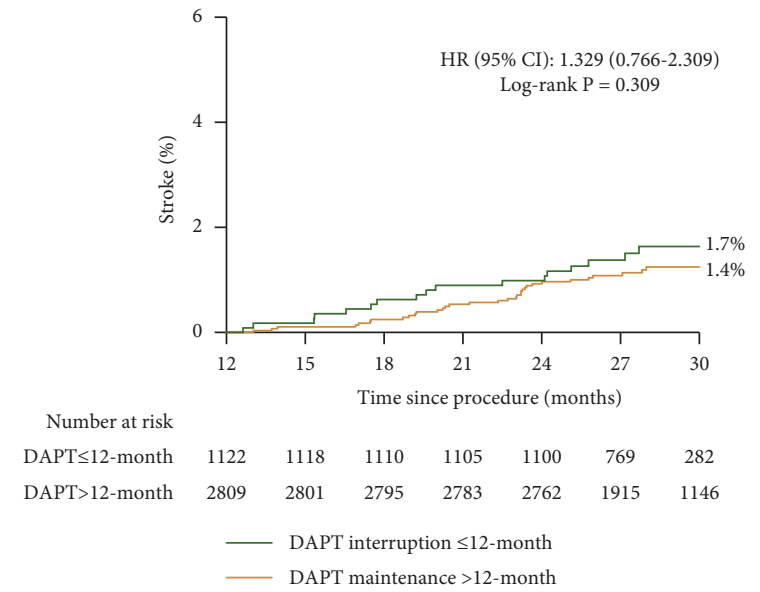

(f)

Figure 3: Continued. 


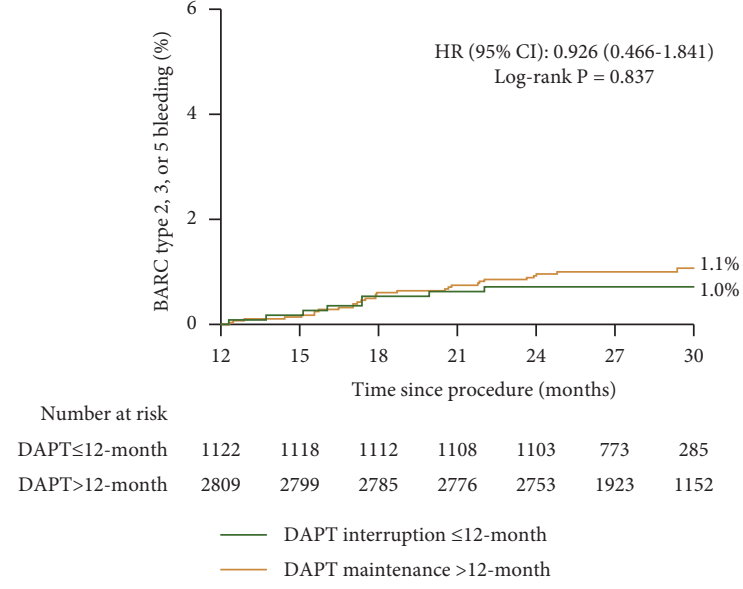

$(\mathrm{g})$

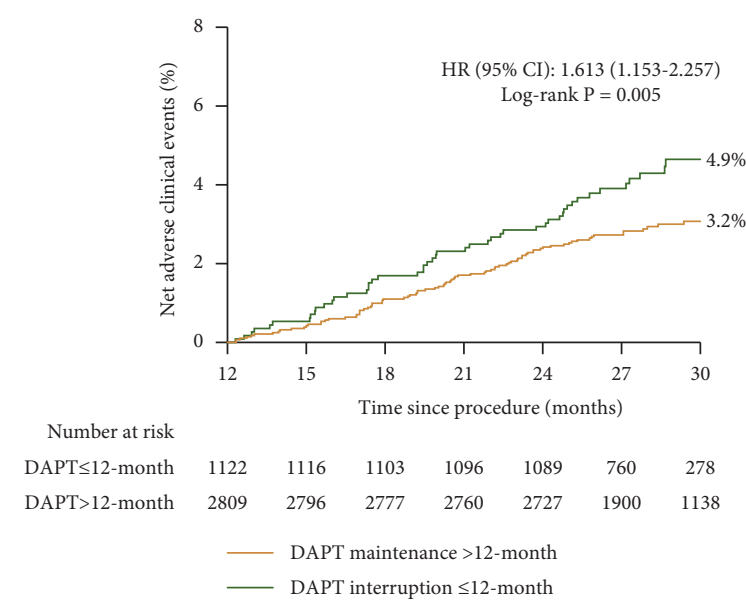

(h)

Figure 3: Time-to-event curves in patients with versus without interruption of DAPT within 12 months. (a) Major adverse cardiac and cerebrovascular events; (b) all-cause death; (c) cardiac death; (d) myocardial infarction; (e) stent thrombosis; (f) stroke; (g) BARC type 2, 3, or 5 bleeding; and (h) net adverse clinical events. Numbers at risk are shown below the chart.

basis (adjusted HR: 1.706, 95\% CI: 1.133-2.596; $P=0.111$ ) (Supplementary Figure 1(b)).

To evaluate the differential effects of DAPT interruption $\leq 12$ months compared with prolonged treatment with DAPT beyond 12 months for various high ischemic risk features according to the 2017 ESC updates for DAPT guidelines, we additionally performed a subgroup analysis according to the components of 2017 ESC high ischemic risk definition (Figure 5(a)). The relationship between DAPT interruption $\leq 12$ months and primary ischemic endpoint was consistent across various clinical or angiographic subsets of high ischemic risk factors. The greatest increased risk in primary ischemic endpoint associated with interruption of DAPT within 12 months was found in patients with diffuse multivessel diabetic CAD (HR: 2.48; 95\% CI: $1.40-4.38 ; P=0.002)$. Relative treatment effects of DAPT interruption $\leq 12$ months were consistent independent of the progressive number of high ischemic risk criteria fulfilled (Figure 5(b)).

We calculated the results of the subgroup analyses comparing the association between DAPT cessation within 12 months and major adverse cardiac and cerebrovascular events for each key subgroup (Table 4 and Supplementary Table 8). In general, the associations were similar in direction and magnitude across key subgroups, and results of formal interaction testing were not significant.

\section{Discussion}

To our knowledge, the present analysis is the first study to date to examine the efficacy and safety of DAPT interruption $\leq 12$ months versus DAPT maintenance $>12$ months after PCI in real-world patients at stent-driven high ischemic risk criteria undergoing PCI with DES, using data from a contemporary prospective cohort. The principal findings of this study are as follows: (1) interruption (temporary or permanent) with the prescribed 1 year of DAPT $(28.5 \%)$ was frequent within 12 months of stent implantation, in which the most common reason of DAPT interruption was bleeding or noncompliance; (2) DAPT interruption significantly increased the risk of primary ischemic endpoint (including death, MI, or stroke) up to 30 months; and (3) there was also a modest but statistically significant increase in 30-month net adverse clinical events in those who discontinued DAPT prematurely. Importantly, the main driver of the difference was an increase in all-cause death and MI without resulting in fewer bleeding events. Taken together, patients who stop DAPT prematurely may require more intensive surveillance to prevent long-term adverse events. The current study provided evidence favoring extendedterm DAPT therapy for ischemic events, which may be appropriate for certain patients who are at a higher risk of cardiovascular events after PCI and low risk of bleeding, such as those presenting with stent-driven high ischemic risk criteria.

Prescribers of DAPT are confronted with a number of challenges for optimal clinical decision making of DAPT type and duration with the scope of minimizing the risk of ischemic and bleeding events in light of each patient's atherothrombotic and hemorrhagic risk and clinical characteristic and circumstance $[1,2]$. Although previous studies showed no significant differences in antithrombotic efficacy between short- and long-term DAPT, longer DAPT treatment was associated with an increased risk for bleeding [6-9]. In most studies, patients had a relatively low risk of recurrent ischemia (mostly patients with chronic coronary syndrome or low-risk ACS). However, whether this short DAPT protects sufficiently against ischemic events and adequately reduces bleeding events among patients at high risk of future stent-driven ischemic events is still unclear because of limited statistical power of the individual trials and mixed results [23]. Of note, evidence regarding decisions about the duration of DAPT for patients at high ischemic risk undergoing PCI in real-world clinical practice is scarce. To address this complex issue, we analyzed the risk of major adverse cardiac and cerebrovascular events in subjects 

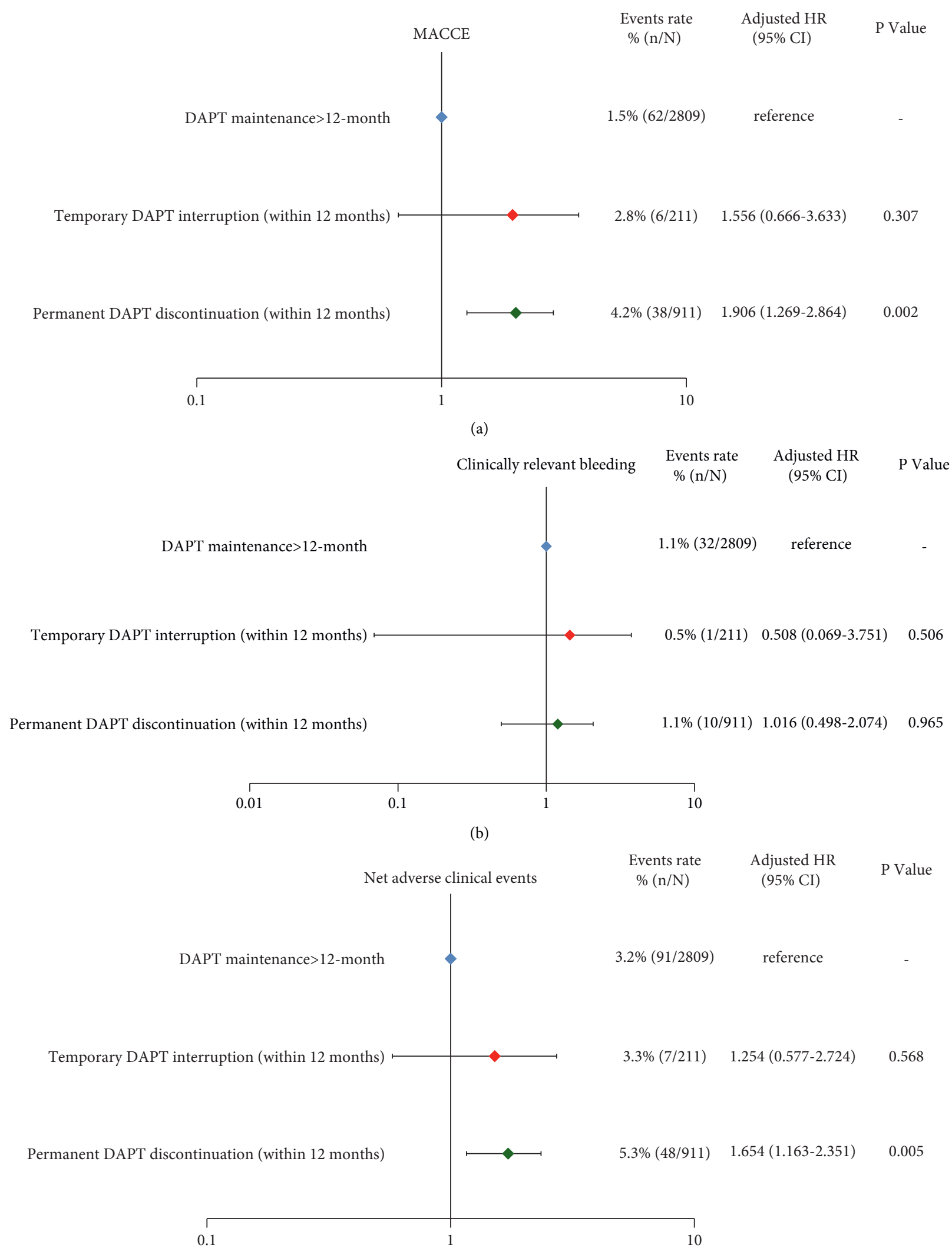

(c)

FIGURE 4: The differential effect of temporary or permanent DAPT interruption within the first 12 months after PCI compared with DAPT maintenance $>12$ months on primary ischemic and key secondary endpoints. MACCE $=$ major adverse cardiac and cerebrovascular events. 


\begin{tabular}{|c|c|c|c|c|c|c|c|}
\hline & Total population & $\begin{array}{l}\text { DAPT interruption } \\
\leq 12-\text { month }(\mathrm{n}=1122)\end{array}$ & $\begin{array}{l}\text { DAPT maintenance } \\
>12-\text { month }(\mathrm{n}=2809)\end{array}$ & & & $\operatorname{HR}(95 \% \mathrm{CI})$ & $P$ value \\
\hline Diffuse multivessel diabetic CAD & $48 / 1673(2.9 \%)$ & $23 / 479(4.8 \%)$ & $25 / 1194(2.1 \%)$ & & $\longmapsto$ & $2.48(1.40-4.38)$ & 0.002 \\
\hline Chronic kidney disease & $24 / 352(6.8 \%)$ & $10 / 104(9.6 \%)$ & $14 / 248(5.6 \%)$ & 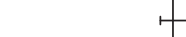 & $\longrightarrow$ & $1.75(0.78-3.94)$ & 0.176 \\
\hline At least 3 stents implanted & $56 / 2107(2.7 \%)$ & $24 / 590(4.1 \%)$ & $32 / 1517(2.1 \%)$ & & $\mapsto$ & $2.05(1.21-3.49)$ & 0.008 \\
\hline At least 3 lesions treated & $16 / 647(2.5 \%)$ & $6 / 189(3.2 \%)$ & $10 / 458(2.2 \%)$ & $\longmapsto$ & - & $1.46(0.53-4.06)$ & 0.469 \\
\hline Bifurcation with 2 stents implanted & $7 / 381(1.8 \%)$ & $2 / 98(2.0 \%)$ & $5 / 283(1.8 \%)$ & & 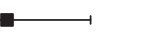 & $1.32(0.25-6.88)$ & 0.511 \\
\hline Total stent length $>60 \mathrm{~mm}$ & $46 / 1811(2.5 \%)$ & $21 / 523(4.0 \%)$ & $25 / 1288(1.9 \%)$ & & $\longmapsto$ & $2.24(1.25-4.01)$ & 0.007 \\
\hline Treatment of chronic total occlusion & $20 / 735(2.7 \%)$ & $9 / 194(4.6 \%)$ & $11 / 541(2.0 \%)$ & & $\longrightarrow$ & $2.47(1.02-5.96)$ & 0.045 \\
\hline \multirow[t]{3}{*}{ Ove rall } & $106 / 3931(2.7 \%)$ & $44 / 1122(3.9 \%)$ & $62 / 2809(2.2 \%)$ & & $1-1$ & $1.96(1.33-2.89)$ & 0.001 \\
\hline & & & & 0.1 & 10.0 & & \\
\hline & & & & DAPT $>12$-month & $\longrightarrow$ & & \\
\hline
\end{tabular}

(a)

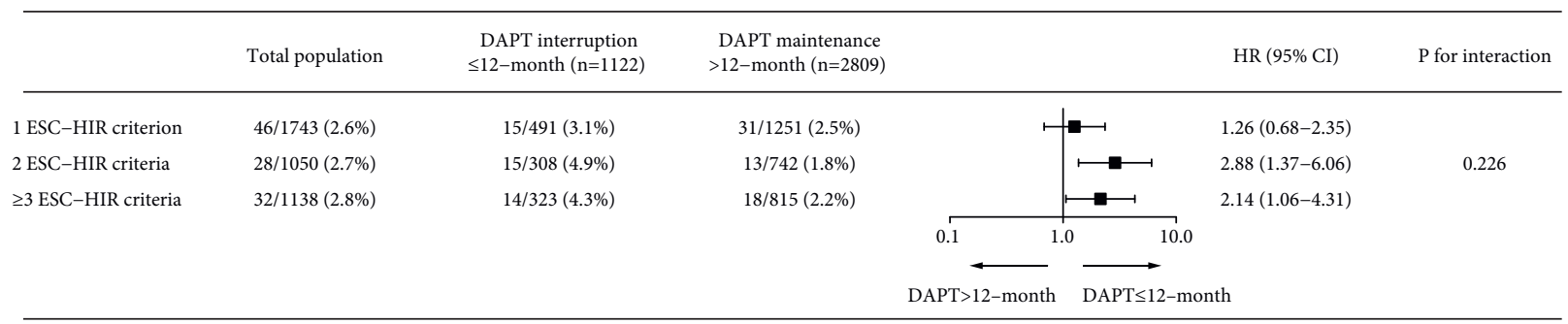

(b)

FIGURE 5: Comparison of long-term risk of primary ischemic endpoint between DAPT interruption $\leq 12$ months and DAPT maintenance $>12$ months according to subgroups. The cumulative incidence and hazard ratio with $95 \%$ confidence interval of primary efficacy endpoint are presented between DAPT $>12$ months and DAPT $\leq 12$ months according to the components of the ESC stent-driven high ischemic risk definition (a) and number of ESC stent-driven high ischemic risk criteria fulfilled (b). CAD = coronary artery disease; $\mathrm{HIR}=$ high ischemic risk; and other abbreviations are as in Figure 1.

who interrupt temporarily or permanently DAPT in the first 12 months after PCI and in patients who met criteria for ESC high ischemic risk and were not at high bleeding risk.

In our cohort, the rate of any interruption of DAPT was $28.5 \%$ at 12 months after PCI, which was in line with the post hoc analysis of ADAPT-DES and other registries that assessed the incidence and effect of DAPT cessation on subsequent cardiovascular risk among patients who underwent PCI with DES implantation [24]. A higher incidence of DAPT cessation (30.2\%) through 1 year of follow-up has been reported in patients undergoing extensive and more complex PCI in the ADAPT-DES registry [25]. Similarly, any nonadherence to DAPT occurred frequently in the contemporary PCI setting, ranging from $5.1 \%$ within 6 months after coronary stents in the DAPT study [12] and 9.6\% during the first 6 months after second-generation DES placement in the EDUCATE registry [13] to $23.3 \%$ over 1 year of follow-up for patients undergoing PCI in the PARIS registry [15] and $44.0 \%$ in patients who discontinued DAPT prematurely $(\leq 12$-month) in the Veterans Affairs healthcare system [24].

Our findings extended insights from the ongoing debate regarding the timing and risk of DAPT interruption in patients treated with current-generation DES. Although short-term to midterm ( $\leq 6$ months) DAPT had similar safety and effectiveness in comparison with 12-month DAPT and better safety than extended-term DAPT, however, these strategies had a higher risk of $\mathrm{MI}$ and stent thrombosis than extended-term DAPT, in turn raising concern about broad application of this practice in higherrisk routine population [26]. We enrolled patients at stentdriven high ischemic risk criteria that were more akin to real-world PCI practice patterns and showed higher longterm risks of cardiac mortality and ischemic events after PCI among patients who had DAPT cessation within 12 months, and these risks persisted even in patients who were free of adverse events in the first year. Recently, Sorrentino et al. [27] demonstrated that disruption of DAPT due to bleeding or poor compliance was associated with an increased risk of major adverse cardiac events and MI at 2 years in patients with history of MI, stroke, or peripheral artery disease. It is important to note that the problem of DAPT nonadherence remains inconclusive for the first 6 months after DES placement. Data from previous studies also showed that discontinuation of DAPT within 6 months of stenting was associated with significantly higher risk of thrombotic complications, including death, MI, and stent thrombosis $[12,13,28]$. Our registry amplified these findings by identifying an independent association of major adverse cardiac events with DAPT interruption $\leq 12$ months, reflecting that physicians appropriately continue DAPT beyond 12 months in high-ischemic risk patients, thereby accounting for lower ischemic events after adopting an extended DAPT strategy. 
Table 4: Primary ischemic endpoint in selected subgroups.

\begin{tabular}{|c|c|c|c|c|}
\hline & $\begin{array}{l}\text { DAPT interruption } \leq 12 \text { months } \\
(n=1122)\end{array}$ & $\begin{array}{l}\text { DAPT maintenance }>12 \text { months } \\
\qquad(n=2809)\end{array}$ & HR (95\% CI) & $\begin{array}{c}P \text { for } \\
\text { interaction }\end{array}$ \\
\hline Age & & & & 0.570 \\
\hline$<65$ years & $21 / 765(2.7 \%)$ & $34 / 1983(1.7 \%)$ & $1.688(0.979-2.912)$ & \\
\hline$\geq 65$ years & $24 / 358(6.7 \%)$ & $27 / 825(3.3 \%)$ & $2.168(1.250-3.762)$ & \\
\hline Sex & & & & 0.470 \\
\hline Female & $12 / 268(4.5 \%)$ & $12 / 654(1.8 \%)$ & $2.454(1.102-5.466)$ & \\
\hline Male & $33 / 855(3.9 \%)$ & $49 / 2154(2.3 \%)$ & $1.829(1.175-2.849)$ & \\
\hline Diabetes mellitus & & & & 0.350 \\
\hline No & $20 / 578(3.5 \%)$ & $33 / 1499(2.2 \%)$ & $1.596(0.914-2.786)$ & \\
\hline Yes & $25 / 545(4.6 \%)$ & $28 / 1309(2.1 \%)$ & $2.346(1.365-4.034)$ & \\
\hline Chronic kidney disease & & & & 0.834 \\
\hline No & $35 / 1019(3.4 \%)$ & $47 / 2560(1.8 \%)$ & $2.026(1.306-3.142)$ & \\
\hline Yes & $10 / 104(9.6 \%)$ & $14 / 248(5.6 \%)$ & $1.751(0.778-3.943)$ & \\
\hline Smoking & & & & 0.182 \\
\hline No & $24 / 495(4.8 \%)$ & $24 / 1208(2.0 \%)$ & $2.577(1.462-4.542)$ & \\
\hline Yes & $21 / 628(3.3 \%)$ & $37 / 1600(2.3 \%)$ & $1.545(0.903-2.644)$ & \\
\hline $\begin{array}{l}\text { Acute coronary } \\
\text { syndrome }\end{array}$ & & & & 0.310 \\
\hline No & $18 / 446(4.0 \%)$ & $22 / 1240(1.8 \%)$ & $2.479(1.326-4.635)$ & \\
\hline Yes & $27 / 677(4.0 \%)$ & $39 / 1568(2.5 \%)$ & $1.632(0.996-2.673)$ & \\
\hline Previous MI & & & & 0.440 \\
\hline No & $31 / 882(4.0 \%)$ & $46 / 2202(2.1 \%)$ & $1.767(1.120-2.788)$ & \\
\hline Yes & $14 / 241(5.8 \%)$ & $15 / 606(2.5 \%)$ & $2.531(1.212-5.287)$ & \\
\hline Multivessel disease & & & & 0.284 \\
\hline No & $6 / 125(4.8 \%)$ & $4 / 283(1.4 \%)$ & $\begin{array}{c}3.683 \\
(1.037-13.083)\end{array}$ & \\
\hline Yes & $39 / 998(3.9 \%)$ & $57 / 2525(2.3 \%)$ & $1.813(1.206-2.728)$ & \\
\hline Generation of DES & & & & 0.889 \\
\hline First-generation DES & $4 / 115(3.5 \%)$ & $6 / 286(2.1 \%)$ & $2.030(0.562-7.340)$ & \\
\hline $\begin{array}{l}\text { Second-generation } \\
\text { DES }\end{array}$ & $41 / 1008(4.1 \%)$ & $55 / 2522(2.2 \%)$ & $1.965(1.310-2.946)$ & \\
\hline ARC-HBR & & & & 0.699 \\
\hline No & $24 / 858(2.8 \%)$ & $39 / 2236(1.7 \%)$ & $1.695(1.018-2.823)$ & \\
\hline Yes & $20 / 264(7.6 \%)$ & $23 / 573(4.0 \%)$ & $1.977(1.085-3.602)$ & \\
\hline
\end{tabular}

DES indicates drug-eluting stent.

Previous studies attempted to evaluate the effect of different modes of DAPT cessation on cardiac events after PCI $[15,29]$. The Xience V coronary stent system trials suggested that the rate of definite and probable ST after cobalt chromium everolimus-eluting stents in patients interrupting DAPT at any point was similar to that of patients who never interrupted DAPT during the 2-year follow-up period, whereas permanent DAPT discontinuation before 3 months was strongly associated with ST in a large, pooled sample of real-world patients [29]. Additionally, Mehran et al. [15] detected no significant increase in thrombotic events in patients who had temporary DAPT interruption lasting up to 14 days. The present study confirms and extends these findings by demonstrating that DAPT interruption on a permanent ( $>30$ days) basis within 1 year after DES implantation was associated with higher 30month rate of major adverse cardiac and cerebrovascular events in patients at increased ischemic risk, while temporary interruption of DAPT did not influence the rate of ischemic events at 30 months. These findings were consistent for patients without experiencing major adverse events of the first 12 months after PCI and for the total study cohort.
A careful assessment of both bleeding and ischemic risks of the individual patient represents the foundation towards personalized medicine in the field of antiplatelet therapy. In the field of managing bleeding vs. ischemic outcomes, we need to optimize therapies for those at HBR after PCI as a post-PCI bleeding event confers an adverse prognosis similar to post-PCI myocardial infarction [30]. Recently, the ARC-HBR criteria were established to standardize the definition of HBR and promote consistency across trials evaluating this vulnerable subset of patients [31]. A number of studies have reported on the predictive value of the ARCHBR definition in identifying patients at increased risk not only for bleeding but also for thrombotic events [18,32-34], as well as validated the clinical usefulness of the ARC-HBR criteria in relation to clinical presentation and sex $[35,36]$. Given that bleeding risk was a major reason for discontinuation of DAPT, we also assessed the effects of DAPT interruption $\leq 12$ months compared with DAPT maintenance $>12$ months in a contemporary PCI population at high ischemic risk and HBR (Table 4). We found that there was no significant difference in the primary ischemic endpoint between treatment arms, irrespective of ARC-HBR 
status. In aggregate, disruption of DAPT was associated with an increased risk of major adverse cardiac events in highischemic risk patients irrespective of the underlying bleeding risk.

In this contemporary cohort of high-risk patients undergoing PCI, the relation between DAPT interruption within 12 months and thrombotic risk is multifactorial and likely both associative and causative in nature. First, in the current analysis, the cumulative incidence of DAPT interruption within 12 months after PCI was higher in ARC-HBR patients, and the higher propensity of HBR patients in developing hemorrhagic complications lead to permanent cessation of DAPT. This finding further emphasizes that DAPT interruption per se may represent a marker of patient risk. Interruption of DAPT in the setting of major bleeding will inevitably result in permanent and abrupt cessation of antithrombotic therapies, need for blood transfusions, invasive procedures to manage bleeding, and their clinical consequences. Second, more and longer stents implanted may increase the likelihood of stent size mismatch, stent underexpansion, malapposition, and overlapping, all of which may act as true mediators of delayed endothelization and enhancing the stent-related thrombotic risk [37]. In the current study, up to one-half of patients received at least 3 stents implanted coexisting with a higher proportion of total stent length $>60 \mathrm{~mm}$, a fact that suggests that encouraged us to prevent premature discontinuation of DAPT and treat them appropriately (e.g., extended DAPT periods). Third, given that patients with multivessel CAD represent an advanced state of atherosclerosis and often leads to incomplete revascularization with a subsequently increased risk of recurrent atherothrombotic coronary events and mortality $[38,39]$, nearly $90 \%$ of the population in our cohort comprised of multivessel CAD and, thus, constitute a high-risk patient group that longer duration of DAPT may be appropriate to mitigate ischemic risks both within and outside of the stented segments. Finally, this large PCI cohort reflecting a real-world setting showed that procedural complexity as assessed by ESC stent-driven high ischemic risk definition adequately captures a patient's cardiovascular and noncardiovascular comorbidities with a greater probability of natural plaque progression followed by future atherothrombotic events, particularly in nontarget lesion events. As reported previously, ischemic events may arise from either stented segments or progressive disease elsewhere in the coronary vasculature $>1$ year after PCI. Late stent-related events were related to patient age, diabetes, and coronary lesion complexity [40]. In this regard, the fatal impact of premature discontinuation of DAPT within 1 year in our dataset might be driven by increasing recurrent ischemic events arising from either from the stented target lesion and nonrevascularized atherosclerotic plaques. Of note in the SWEDEHEART registry, the risk of recurrent MI not originating from a previously stented lesion was twice as high as the risk of lesions originating from a previously stented lesion, emphasizing the importance of preventing atherothrombotic events from nontreated lesions long term and overall coronary disease progression after an initial MI [41]. Collectively, our findings provided additional insights to the expanding evidence base for utilizing 2017 ESC stentdriven high ischemic risk criteria to guide clinical practice to identify patients who might benefit from prolonging antithrombotic treatment duration.

4.1. Limitations. Certain limitations were present with the analyses presented within this study. First, our data were derived from a large volume single-center PCI registry and may suffer from limited generalizability. Second, although the data were collected prospectively, as for any retrospective study, our findings should be considered hypothesis generating, and future trials are needed to confirm our findings. We performed additional sensitivity analyses, but there is a possibility of unmeasured confounding factors that may lead to increased risk of ischemic events associated with subjects who had an interruption or discontinued DAPT. Third, the decision to discontinue or remain on DAPT after 12 months was made at the discretion of the patient's physician (and possibly influenced by the patient); hence, selection bias was inevitable in this specific substudy cohort that might have affected event rates. Fourth, ticagrelor $(0.4 \%)$ only became available late during the study recruitment, and prasugrel was unavailable in China; as a result, most of patients received clopidogrel as a $\mathrm{P}_{2} \mathrm{Y}_{12}$ inhibitor for DAPT. However, owing to the differential propensity for bleeding events with response to antiplatelet therapy, East Asian populations undergoing PCI treated with potent $\mathrm{P}_{2} \mathrm{Y}_{12}$ inhibitors did not have a lower ischemic outcome, but had higher incidence of clinically significant bleeding compared with clopidogrel $[42,43]$. In this scenario, our patients were less prone to bleeding and were more likely to show a net clinical outcome. Furthermore, although CYP2C19 genotyping might be used as an optional tool for guiding antiplatelet therapy, it was not available for this study. Finally, our study was performed in a Chinese population. Compared with Western population, East Asian population has a higher prevalence of the CYP2C19 loss-offunction genotype, which is associated with a higher level of platelet reactivity during clopidogrel treatment. Thrombogenicity, pharmacogenetics, and susceptibility for bleeding complication on P2Y12 inhibitors could be different between Asian and Western population. Thus, we should be cautious about extrapolating these study results outside China.

\section{Conclusions}

In this large-scale PCI cohort of patients with stent-driven high ischemic risk definition, interruption of DAPT (temporary or permanent) predominantly due to poor compliance or bleeding complications within 12 months was associated with significantly higher risk for cardiovascular ischemic recurrences at 30 months compared with prolonged-term (>12 months) DAPT. Patients who had DAPT interruption may benefit from more intensive surveillance to prevent long-term cardiovascular events. Our findings may inform future considerations for utilizing ESC stent-driven high ischemic risk criteria to help clinicians adopting a prolonged DAPT course to pursue a best benefit-risk ratio in an individual patient. 


\section{Data Availability}

The clinical and procedural data used to support the findings of this study are included within the article.

\section{Ethical Approval}

Data collection for this study was approved by the institutional review board central committee at Fuwai Hospital, National Center for Cardiovascular Diseases in China.

\section{Conflicts of Interest}

The authors declare no conflicts of interest regarding the publication of this paper.

\section{Acknowledgments}

This research was supported by the Chinese Academy of Medical Sciences Innovation Fund for Medical Sciences (CIFMS) (Grant no. 2021-I2M-1-008), Beijing Health Promotion Association (Grant no. 2020-ZX52), Chinese College of Cardiovascular Physicians, CS Optimizing Antithrombotic Research Fund (Grant no. BJUHFCSOARF201801-01), and Beijing Municipal Health Commission (Grant no. 2020-1-4032). Hao-Yu Wang would like to express his sincere appreciation to Ran Mo (from the Emergency and Critical Care Center, Fuwai Hospital, Chinese Academy of Medical Sciences and Peking Union Medical College) for her important contributions to this study.

\section{Supplementary Materials}

Supplementary Table 1: study definitions for major and minor ARC-HBR criteria compared with the original definitions from the ARC-HBR document; Supplementary Table 2: percent standardized differences of variables among unadjusted, propensity score matching, and IPTWadjusted cohort; Supplementary Table 3: ischemic and bleeding outcomes according to ESC high ischemic risk criteria status from 12 to 30 months after coronary stent treatment $(n=9212)$; Supplementary Table 4: 30 -month ischemic and bleeding outcomes by DAPT interruption status in the propensity score matching cohort; Supplementary Table 5: baseline characteristics in the entire population $(n=4,430)$; Supplementary Table 6: lesion and procedural characteristics in the entire population $(n=4,430)$; Supplementary Table 7: 30 -month ischemic and bleeding outcomes by DAPT interruption status in the entire population $(n=4,430)$; Supplementary Table 8: primary ischemic endpoint in selected subgroups in the entire population $(n=4,430)$; Supplementary Figure 1: the differential effect of temporary or permanent DAPT interruption within the first 12 months after PCI compared with DAPT maintenance $>12$ months on primary ischemic and key secondary endpoints in the entire population $(n=4,430)$. (Supplementary Materials)

\section{References}

[1] D. Cao, R. Chandiramani, M. Chiarito, B. E. Claessen, and R. Mehran, "Evolution of antithrombotic therapy in patients undergoing percutaneous coronary intervention: a 40-year journey," European Heart Journal, vol. 42, no. 4, pp. 339-351, 2021.

[2] G. N. Levine and E. R. Bates, "It is time to end the dualistic short versus long duration of dual antiplatelet therapy debates," Circulation, vol. 135, no. 25, pp. 2451-2453, 2017.

[3] M. Valgimigli, H. Bueno, R. A. Byrne, J. P. Collet, F. Costa, and A. Jeppsson, "2017 ESC focused update on dual antiplatelet therapy in coronary artery disease developed in collaboration with EACTS: the task force for dual antiplatelet therapy in coronary artery disease of the European Society of Cardiology (ESC) and of the European Association for Cardio-Thoracic Surgery (EACTS)," European Heart Journal, vol. 39, no. 3, pp. 213-260, 2017.

[4] G. Ferrante, G. Condorelli, P. Pagnotta, and B. Reimers, "Dual antiplatelet therapy continuation beyond 1 year after drugeluting stents," Circulation: Cardiovascular Interventions, vol. 10, no. 5, 2017.

[5] G. W. Stone, "Balancing ischaemia and bleeding with dual antiplatelet therapy: a resolute endeavour," European Heart Journal, vol. 35, no. 29, pp. 1914-1916, 2014.

[6] B.-K. Kim, M.-K. Hong, D.-H. Shin et al., "A new strategy for discontinuation of dual antiplatelet therapy," Journal of the American College of Cardiology, vol. 60, no. 15, pp. 1340-1348, 2012.

[7] F. Feres, R. A. Costa, A. Abizaid, M. B. Leon, J. A. Marin-Neto, and R. V. Botelho, "Three vs twelve months of dual antiplatelet therapy after zotarolimus-eluting stents," JAMA, vol. 310, no. 23, pp. 2510-2522, 2013.

[8] M. Valgimigli, G. Campo, M. Monti et al., "Short- versus longterm duration of dual-antiplatelet therapy after coronary stenting," Circulation, vol. 125, no. 16, pp. 2015-2026, 2012.

[9] M. Gilard, P. Barragan, A. A. L. Noryani et al., "6- versus 24month dual antiplatelet therapy after implantation of drugeluting stents in patients nonresistant to aspirin," Journal of the American College of Cardiology, vol. 65, no. 8, pp. 777-786, 2015.

[10] M. Valgimigli, F. Gragnano, M. Branca et al., "P2Y12 inhibitor monotherapy or dual antiplatelet therapy after coronary revascularisation: individual patient level meta-analysis of randomised controlled trials," BMJ, vol. 373, p. 1332, 2021.

[11] C. Yerasi, B. C. Case, B. J. Forrestal et al., "Optimizing monotherapy selection, aspirin versus P2Y12 inhibitors, following percutaneous coronary intervention," The American Journal of Cardiology, vol. 135, pp. 154-165, 2020.

[12] A. C. Stefanescu Schmidt, P. G. Steg, R. W. Yeh et al., "Interruption of dual antiplatelet therapy within six months after coronary stents (from the dual antiplatelet therapy study)," The American Journal of Cardiology, vol. 124, no. 12, pp. 1813-1820, 2019.

[13] D. E. Cutlip, D. J. Kereiakes, L. Mauri, R. Stoler, and H. L. Dauerman, "Thrombotic complications associated with early and late nonadherence to dual antiplatelet therapy," JACC: Cardiovascular Interventions, vol. 8, no. 3, pp. 404-410, 2015.

[14] S. Silber, A. J. Kirtane, J. A. Belardi et al., "Lack of association between dual antiplatelet therapy use and stent thrombosis between 1 and 12 months following resolute zotarolimuseluting stent implantation," European Heart Journal, vol. 35, no. 29, pp. 1949-1956, 2014. 
[15] R. Mehran, U. Baber, P. G. Steg et al., "Cessation of dual antiplatelet treatment and cardiac events after percutaneous coronary intervention (PARIS): 2 year results from a prospective observational study," The Lancet, vol. 382, no. 9906, pp. 1714-1722, 2013.

[16] F. J. Neumann, M. Sousa-Uva, A. Ahlsson, F. Alfonso, A. P. Banning, and U. Benedetto, "2018 ESC/EACTS guidelines on myocardial revascularization," European Heart Journal, vol. 40, no. 2, pp. 87-165, 2018.

[17] G. N. Levine, E. R. Bates, J. C. Blankenship et al., “2011 ACCF/AHA/SCAI guideline for percutaneous coronary intervention," Journal of the American College of Cardiology, vol. 58, no. 24, pp. e44-e122, 2011.

[18] N. Corpataux, A. Spirito, F. Gragnano et al., "Validation of high bleeding risk criteria and definition as proposed by the academic research consortium for high bleeding risk," $E$ uropean Heart Journal, vol. 41, no. 38, pp. 3743-3749, 2020.

[19] R. Mehran, S. V. Rao, D. L. Bhatt et al., "Standardized bleeding definitions for cardiovascular clinical trials," Circulation, vol. 123, no. 23, pp. 2736-2747, 2011.

[20] D. E. Cutlip, S. Windecker, R. Mehran et al., "Clinical end points in coronary stent trials," Circulation, vol. 115, no. 17, pp. 2344-2351, 2007.

[21] K. Thygesen, J. S. Alpert, A. S. Jaffe et al., "Third universal definition of myocardial infarction," Journal of the American College of Cardiology, vol. 60, no. 16, pp. 1581-1598, 2012.

[22] R. L. Sacco, S. E. Kasner, J. P. Broderick et al., "An updated definition of stroke for the 21 st century," Stroke, vol. 44, no. 7, pp. 2064-2089, 2013.

[23] R. Mehran, G. Giustino, and U. Baber, "DAPT duration after DES," Journal of the American College of Cardiology, vol. 65, no. 11, pp. 1103-1106, 2015.

[24] S. Kinlay, L. Quach, J. Cormack et al., "Premature discontinuation of dual antiplatelet therapy after coronary stenting in veterans: characteristics and long-term outcomes," Journal of American Heart Association, vol. 10, no. 9, Article ID e018481, 2021.

[25] P. Généreux, G. Giustino, B. Redfors et al., "Impact of percutaneous coronary intervention extent, complexity and platelet reactivity on outcomes after drug-eluting stent implantation," International Journal of Cardiology, vol. 268, pp. 61-67, 2018.

[26] S. U. Khan, M. Singh, S. Valavoor et al., "Dual antiplatelet therapy after percutaneous coronary intervention and drugeluting stents," Circulation, vol. 142, no. 15, pp. 1425-1436, 2020.

[27] S. Sorrentino, G. Giustino, U. Baber et al., "Dual antiplatelet therapy cessation and adverse events after drug-eluting stent implantation in patients at high risk for atherothrombosis (from the PARIS registry)," The American Journal of Cardiology, vol. 122, no. 10, pp. 1638-1646, 2018.

[28] F. Airoldi, A. Colombo, N. Morici et al., "Incidence and predictors of drug-eluting stent thrombosis during and after discontinuation of thienopyridine treatment," Circulation, vol. 116, no. 7, pp. 745-754, 2007.

[29] P. Généreux, D. R. Rutledge, T. Palmerini et al., "Stent thrombosis and dual antiplatelet therapy interruption with everolimus-eluting stents," Circulation: Cardiovascular Interventions, vol. 8, no. 5, 2015.

[30] S. Leonardi, F. Gragnano, G. Carrara et al., "Prognostic implications of declining hemoglobin content in patients hospitalized with acute coronary syndromes," Journal of the American College of Cardiology, vol. 77, no. 4, pp. 375-388, 2021.
[31] P. Urban, R. Mehran, R. Colleran et al., "Defining high bleeding risk in patients undergoing percutaneous coronary intervention," Circulation, vol. 140, no. 3, pp. 240-261, 2019.

[32] Y. Ueki, S. Bär, S. Losdat et al., "Validation of the Academic Research Consortium for High Bleeding Risk (ARC-HBR) criteria in patients undergoing percutaneous coronary intervention and comparison with contemporary bleeding risk scores," EuroIntervention, vol. 16, no. 5, pp. 371-379, 2020.

[33] D. Cao, R. Mehran, G. Dangas et al., "Validation of the academic research consortium high bleeding risk definition in contemporary PCI patients," Journal of the American College of Cardiology, vol. 75, no. 21, pp. 2711-2722, 2020.

[34] M. Natsuaki, T. Morimoto, H. Shiomi et al., "Application of the academic research consortium high bleeding risk criteria in an all-comers registry of percutaneous coronary intervention," Circulation: Cardiovascular Interventions, vol. 12, no. 11, Article ID e008307, 2019.

[35] F. Gragnano, A. Spirito, N. Corpataux et al., "Impact of clinical presentation on bleeding risk after percutaneous coronary intervention and implications for the ARC-HBR definition," EuroIntervention, vol. 17, no. 11, pp. e898-e909, 2021.

[36] A. Spirito, F. Gragnano, N. Corpataux et al., "Sex-based differences in bleeding risk after percutaneous coronary intervention and implications for the academic research consortium high bleeding risk criteria," Journal of American Heart Association, vol. 10, no. 12, Article ID e021965, 2021.

[37] G. G. Stefanini and D. R. Holmes Jr., "Drug-eluting coronaryartery stents," New England Journal of Medicine, vol. 368, no. 3, pp. 254-265, 2013.

[38] P. Sorajja, B. J. Gersh, D. A. Cox et al., "Impact of multivessel disease on reperfusion success and clinical outcomes in patients undergoing primary percutaneous coronary intervention for acute myocardial infarction," European Heart Journal, vol. 28, no. 14, pp. 1709-1716, 2007.

[39] R. Piccolo, G. Giustino, R. Mehran, and S. Windecker, "Stable coronary artery disease: revascularisation and invasive strategies," The Lancet, vol. 386, no. 9994, pp. 702-713, 2015.

[40] M. V. Madhavan, A. J. Kirtane, B. Redfors et al., "Stent-related adverse events $>1$ year after percutaneous coronary intervention," Journal of the American College of Cardiology, vol. 75, no. 6, pp. 590-604, 2020.

[41] C. Varenhorst, P. Hasvold, S. Johansson et al., "Culprit and nonculprit recurrent ischemic events in patients with myocardial infarction: data from SWEDEHEART (Swedish web system for enhancement and development of evidence-based care in heart disease evaluated according to recommended therapies)," Journal of American Heart Association, vol. 7, no. 1, Article ID e007174, 2018.

[42] J. Kang, J.-K. Han, Y. Ahn et al., "Third-generation P2Y12 inhibitors in East Asian acute myocardial infarction patients: a nationwide prospective multicentre study," Thrombosis \& Haemostasis, vol. 118, no. 03, pp. 591-600, 2018.

[43] H. Cho, J. Kang, H.-S. Kim, and K. W. Park, "Ethnic differences in oral antithrombotic therapy," Korean Circulation Journal, vol. 50, no. 8, pp. 645-657, 2020. 